\title{
Time-Reversal Invariant Topological Superconductivity in Quasi-One-Dimensional Structures
}

\author{
S. Mammadova*, E. Nakhmedov, O. Alekperov \\ Institute of Physics, Azerbaijan National Academy of Sciences, H. Cavidave. 33, AZ1143 Baku, Azerbaijan

\begin{abstract}
It is shown that a time-reversal invariant topological superconductivity can be realized in a quasi-onedimensional structure, which is fabricated by filling the superconducting materials into the periodic channel of dielectric matrices like zeolite and asbestos under high pressure. The topological superconducting phase sets up in the presence of large spin-orbit interactions when s-wave intra-wire and d-wave inter-wire pairings take place. Kramers pairs of Majorana bound states emerge at the edges of each wire. The time-reversal topological superconductor belongs to DIII class of symmetry with a $Z_{2}$ invariant.
\end{abstract}

DOI: 10.12693/APhysPolA.129.800

PACS/topics: 74.78.--w, 71.10.Pm, 71.70.Ej, 74.50.+r

\section{Introduction}

The recent theoretical prediction of topological insulators and superconductors [1] and their experimental observation in a number of materials have attracted great interest to this subject. The Hamiltonians for gapped topological insulators and superconductors are classified into ten topological symmetry classes [2, 3] each of which is characterized by a topological invariant. The time-reversal invariant (TRI) topological superconductors (SC) were predicted by theoretical classification of Bogolyubov-de Gennes (BdG) Hamiltonian which constitutes a completely distinct symmetry class DIII. Topological SC has a bulk gap and gapless Andreev bound states. Due to the presence of intrinsic particle-hole symmetry (PHS) the gappless zero-modes in the topological superconductors constitute Majorana fermions (MF), obeying non-Abelian braiding statistics which is useful in implementing fault-tolerant topological quantum computer [4]. The challenge is to find a real material which supports the topological SC properties.

Recent works $[5,6]$ have proposed TRI topological superconductivity (class $D I I I$ ) with a $Z_{2}$ invariant, which takes a value $\nu=1$, in a number of systems with intrinsic or proximity induced superconductivity of p-wave, spintriplet or d-wave and s $\mathrm{s}$-wave spin-singlet pairings.

In this work, it is shown that the time-reversal invariant topological superconducting phase can be realized in quasi-one-dimensional (quasi-1D) wires on the plane in the presence of s-wave intra-wire and d-wave inter-wire pairings. Similar structures have been fabricated $[7,8]$ by filling a superconducting material into the cavities or channels of dielectric matrices like zeolite and asbestos crystals (see, e.g. Fig. 1a) under high pressure up to 30 kbar. The regular set of channels or cavities with diameter of $5-10 \AA$ in zeolite and from $20-30 \AA$ up to

*corresponding author; e-mail: memmedova_seide.fiz@mail.ru
100-150 $\AA$ in asbestos [9] form a periodic lattice of different geometry in one, two and three dimensions, e.g. like several equidistant filaments with $5 \div 20 \AA$ separation in zeolite and with $150 \div 500 \AA$ separation in asbestos on a plane or quasi-1D space lattice. The critical temperatures $T_{\mathrm{c}}$ of such structures become higher than the $T_{\mathrm{c}}$ of the bulk superconductors by factors of 2-5 [7]. High stress field around the filaments may guarantee higher value of spin-orbit interactions in the structures. An increase in the critical temperature may be caused by an additional inter-wire pairing due to polarization of the dielectric matrix between the wires.

\section{Time reversal invariant topological superconductor}

The equidistant superconducting wires, aligned along $\mathrm{x}$-axes in $\{\mathrm{x}, \mathrm{y}\}$ plane, with intra- and inter-wire s-wave pairings (see, Fig. 1b), in the presence of spin-orbit interactions (SOIs) are described in the momentum space by Hamiltonian

$$
\hat{H}=\int_{-\pi}^{\pi} \int_{-\pi}^{\pi} \frac{d k_{x} d k_{y}}{(2 \pi)^{2}} \psi_{k}^{+} H \psi_{k},
$$

in the extended Nambu spinor basis $\psi_{k}^{+}=$ $\left(\psi_{k, \uparrow}^{+}, \psi_{k, \downarrow}^{+}, \psi_{-k, \downarrow},-\psi_{-k, \uparrow}\right) . H$ in Eq. 1 reads as

$$
\begin{aligned}
& H=\xi_{k} \tau_{z}+2\left(\alpha \sin k_{x}-\bar{\beta} \sin k_{y}\right) \tau_{z} \sigma_{y} \\
& \quad+2\left(\beta \sin k_{x}-\bar{\alpha} \sin k_{y}\right) \tau_{z} \sigma_{x}+\Delta\left(k_{y}\right) \tau_{x},
\end{aligned}
$$

where, $\xi_{k}=-2 t_{\|} \cos k_{x}-2 t_{\perp} \cos k_{y}-\mu$ is the energy dispersion in a single wire, $t_{\|},\left(t_{\perp}\right)$ and $\mu$ are the longitudinal (transverse) overlap integral and the Fermi energy, $\alpha(\beta)$ and $\bar{\alpha}(\bar{\beta})$ are the longitudinal and transverse components of Rashba (Dresselhaus) spin-orbit constants. The existence of s-wave intra-wire and d-wave inter-wire order parameters $\Delta_{0}$ and $\Delta_{1}$, which are assumed here to be real parameters, allows one to introduce the effective order parameter $\Delta\left(k_{y}\right)$ in momentum space, $\Delta\left(k_{y}\right)=\Delta_{0}+2 \Delta_{1} \cos k_{y}$. The Pauli matrices $\sigma_{i}$ and $\tau_{i}$ in Eq. 2 act in the spin- and particle-hole spaces, 
respectively. We assume also that the structure is strongly anisotropic and the condition $t_{\perp} \ll t_{\|}$is satisfied. The Fermi surface under this condition becomes opened, consisting of two goffered lines. Note, that a conventional Josephson coupling between the wires is realized under $t_{\perp}<k_{\mathrm{B}} T_{\mathrm{c} 0} \ll t_{\|}$, where $T_{\mathrm{c} 0}$ is the critical temperature of the superconducting phase evaluated within the mean field theory. The time-reversal symmetry and the particle-hole symmetry are protected in topological SC. Hamiltonian $H$ satisfies the relations $\Theta H_{0}(k)=H_{0}(-k) \Theta$ with TRI operator $\Theta=i \sigma_{y} K$, where $K$ is the anti-unitary complex conjugation operator. The particle-hole symmetry emerges from the intrinsic structure of the BdG Hamiltonian, which satisfies the anti-commutation relation $\Xi H_{0}=-H_{0} \Xi$ with the particle-hole operator $\Xi=\tau_{y} \sigma_{y} K$, obeying $\Xi=-1$. The presence of time-reversal symmetry and particle-hole symmetry leads to a chiral symmetry $\Pi H(k)=-H(-k) \Pi$ with the unitary chiral operator $\Pi=-i \Theta \Xi=\tau_{y} \sigma_{0}$.
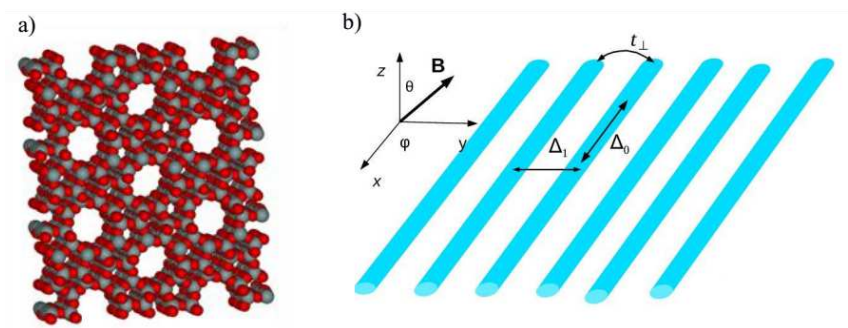

Fig. 1. Structure of (a) a porous zeolite with periodic channels, and (b) the corresponding model for the quasi1D superconducting wires on a plane.

The energy spectrum, obtained from det $\left|E_{0}-H_{0}\right|=0$, reads

$$
E_{0}=s \sqrt{\left(\xi_{k}^{2} \pm \varepsilon_{s}\right)^{2}+\left|\Delta_{\text {eff }}\left(k_{y}\right)\right|^{2}} .
$$

Here $s= \pm$ and $\varepsilon_{s}=\left[\left(\sin ^{2} k_{x}+\sin ^{2} k_{y}\right)\left(\alpha^{2}+\beta^{2}\right)-\right.$ $\left.4 \alpha \beta \sin k_{x} \sin k_{y}\right]^{1 / 2}$ (we take $\alpha=\bar{\alpha}$ and $\beta=\bar{\beta}$ for simplicity). $\Delta\left(k_{y}\right)=\Delta_{0}+2 \Delta_{1} \cos k_{y}$ changes sign at the nodal point $\cos k_{y}=\mp \frac{\Delta_{0}}{2 \Delta_{1}}$ if $\left|\Delta_{0}\right|<2 \Delta_{1}$ while moving along $k_{y}$ from $k_{y}=0$ to $k_{y}=\pi$. A nontrivial TRI superconductor with $\nu=1$ is realized if there is an odd number of Fermi surfaces with a negative pairing order parameter [3]. At the nodal points one gets $E_{0 \mathrm{~N}}=s\left[2 t_{\|} \cos k_{x}+\mu-t_{\perp} \frac{\Delta_{0}}{2 \Delta_{1}} \pm E_{\mathrm{SN}}\left(k_{x}, k_{y N}\right)\right]$ from Eq. 3, where $\delta=\sqrt{1-\frac{\Delta_{0}^{2}}{\Delta_{1}^{2}}}$ and $E_{\mathrm{SN}}\left(k_{x}, k_{y N}\right)$ is the value of the SOI energy $E_{\mathrm{SN}}$ at the nodal point $k_{y N}=\arccos \left(-\frac{\Delta_{0}}{2 \Delta_{1}}\right)$, $E_{\mathrm{SN}}\left(k_{x}, k_{y N}\right)=\left[\left(\sin ^{2} k_{x}+\delta^{2}\right)\left(\alpha^{2}+\beta^{2}\right)-4 \alpha \beta \delta \sin k_{x}\right]^{1 / 2}$. $E_{\mathrm{SN}}\left(k_{x}, k_{y N}\right)$ varies between the maximal $E_{S N}^{\max }$ and minimal $E_{S N}^{\min }$ values. The order parameter switches sign as the nodal point is crossed. On the other hand, the SOIs split the Fermi surfaces. The splitted Fermi surfaces around the nodal points lie in the energy interval of $E_{\mathrm{SN}}\left(k_{x}, k_{y N}\right)$ from each other. Non-trivial TRI topological phase with $\nu=1$ is realized when the maximal value of the kinetic energy term (the first three terms in $\mathrm{E}_{0 N}$ ) is smaller than the minimal value of the SOIs mediated splitting energy $E_{\mathrm{SN}}\left(k_{x}, k_{y N}, \alpha, \beta\right),\left|2 t_{\|}+\mu-t_{\perp} \frac{\Delta_{0}}{\Delta_{1}}\right|<$ $E_{\mathrm{SN}}^{\min }\left(k_{x 0}, \alpha, \beta\right)$.

The SC is fully gapped when $\left|2 t_{\|}+\mu-t_{\perp} \frac{\Delta_{0}}{\Delta_{1}}\right|>$ $E_{\mathrm{SN}}^{\max }\left(k_{x 0}, \alpha, \beta\right)$. The calculation of the $\mathrm{BdG}$ quasiparticles' energy spectrum is simplified for $\beta=0$, for which $E_{\mathrm{SN}}^{\min }\left(k_{x 0}, \alpha, \beta\right)=2 \alpha \delta$ and $E_{\mathrm{SN}}^{\max }\left(k_{x 0}, \alpha, \beta\right)=$ $2 \alpha \sqrt{1+\delta^{2}}$. The band structure of the topological SC with zero energy surface states for this case is drawn in Figs. 2a,b. For $\Delta_{0}<0$, Majorana edge states appear at $k_{x}=0$, which are shown in Fig. 2a by red curves. The zero energy states move to the Brillouin zone boundaries, $k_{x}= \pm \pi$, for $\Delta_{0}>0$ (see Fig. 2b). Even though a pair of zero energy states are localized at the same end, they are protected by time-reversal symmetry against hybridization, preventing their splitting to finite energies.
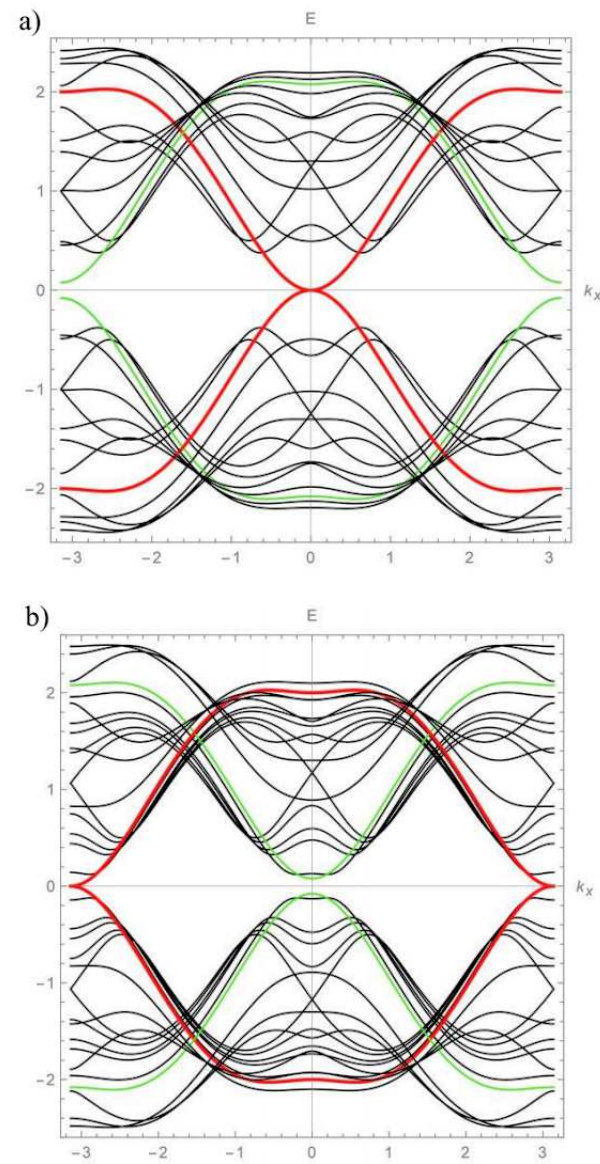

Fig. 2. The energy spectrum of quasi-1D superconductor as a function of $k_{x}$ in the non-trivial topological phase $(\nu=1)$ for (a) $\Delta_{0}>0$ with a zero-energy state (red curves) at the center of the Brillouin zone and (b) $\Delta_{0}<0$ with a zero energy state (red curves) at the boundaries of the Brillouin zone. The parameters are chosen in the unit of the band width $2 t_{\|}=1$. In both cases $\left|\Delta_{0}\right|=0.5 \times 2 t_{\|}$we choose, $t_{\perp}=0.2 \times 2 t_{\|}$and $\alpha=0.6 \times 2 t_{\|}$. 


\section{Conclusion}

In this paper it is argued that a TRI topological superconducting phase may be realized in the novel class of materials, consisting of regular, weakly-coupled superconducting wires in dielectric matrices [7-9]. The structures are fabricated under high pressures, which guarantee higher value of spin-orbit interactions. Experimentally observed enhancement of the critical temperature of these structures allows us to suggest that apart from the intra-wire pairing, the inter-wire pairing sets up too, yielding an effective nodal order parameter. The order parameter changes sign by crossing the nodal point between two Fermi surfaces, splitted due to spin-orbit interactions. Note, that the only requirement for realization of a non-trivial topological superconductor is that the superconducting pair potential switches sign between the two Fermi surfaces. Time-reversal symmetric topological superconductor belongs to a DIII symmetry-class and is classified by the $D I I I Z_{2}$ topological invariant.

\section{Acknowledgments}

S.M. kindly acknowledges support from Research Grant EIF-2012-2(6)-39/08/1 of the Science Development Foundation under the President of the Republic of Azerbaijan.

\section{References}

[1] M.Z. Hasan, C.L. Kane, Rev. Mod. Phys. 82, 3045 (2010).

[2] A.P. Schnyder, S. Ryu, A. Furusaki, A.W.W. Ludwig, Phys. Rev. B 78, 195125 (2008).

[3] X.L. Qi, T.L. Hughes, S.C. Zhang, Phys. Rev. B 81, 134508 (2010).

[4] C. Nayak, S.H. Simon, A. Stern, M. Freedman, S. Das Sarma, Rev. Mod. Phys. 80, 1083 (2008).

[5] F. Zhang, C.L. Kane, E.J. Mele, Phys. Rev. Lett. 111, 056402 (2013).

[6] C.L.M. Wong, K.T. Law, Phys. Rev. B 86, 184516 (2012).

[7] V.N. Bogomolov, Fiz. Tverd. Tela (Leningrad) 13 , 815 (1971); ibidem 15, 1312 (1973); Sov. Phys. Solid State 13, 672 (1971); ibidem 15, 894 (1973).

[8] V.N. Bogomolov, Yu.A. Kumzerov, Pis'ma v Zh.. Eksp. Teor. Fiz. 21, 434 (1975); JETP Lett. 21, 198 (1975).

[9] V.N. Bogomolov, Sov. Phys. Usp. 21, 77 (1978); Usp. Fiz. Nauk 124, 171 (1978). 